\title{
The significance of arterial hypertension at the onset of clinical lupus nephritis
}

\author{
Indiran P Naiker, Vivien Chrystal, Ismail GH Randeree, Yackoob K Seedat
}

\begin{abstract}
Summary
The prognostic importance of hypertension at the onset of clinical lupus nephritis is not well established. We studied retrospectively 44 patients with lupus nephritis in order to ascertain the prevalence of hypertension at presentation and to investigate a possible association between hypertension and renal functional impairment. A correlation was also sought between hypertension and histological class of lupus nephritis. Hypertension was graded as mild (diastolic 95-99 mmHg), moderate (100-114) or severe ( $>115$ ). Impaired renal function (creatinine $>120 \mu \mathrm{mol} / \mathrm{l})$ was graded as mild (120-200 $\mu \mathrm{mol} / 1)$, moderate (200-350 $\mu \mathrm{mol} / \mathrm{l})$, or severe $(>350 \mu \mathrm{mol} / \mathrm{l})$. Histological class and the presence of hypertensive renal vascular lesions was recorded.

The prevalence of hypertension was $38 \%$. There were 17 hypertensives and 27 normotensives. The incidence of renal impairment was greater in the hypertensives, $47 \%$ vs $18.5 \%(p=0.04)$. Mean serum creatinine was also higher higher in this group $(p=0.02)$. The presence of hypertensive renal vascular lesions identified a high-risk subgroup who had a higher incidence of renal functional impairment and worse renal function than the hypertensive group as a whole. Even at an early stage, hypertension and hypertensive renal vascular lesions correlated well with renal functional impairment. Aggressive treatment of hypertension is therefore essential in early lupus nephritis in order to prevent further deterioration of renal function as the disease evolves.
\end{abstract}

University of Natal

Edward VIII

Hospital, Durban,

South Africa

Department of

Medicine

IP Naiker

YK Seedat

IGH Randeree

Department of

Anatomical

Pathology

V Chrystal

Correspondence to

Dr Indiran P Naiker,

Department of Medicine, Medical School,

University of Natal, PO

Box 17039, Congella,

Box 17039, Congella,
4013, Durban, South

Africa

Accepted 27 June 1996

The development of arterial hypertension patients with systemic lupus erythematosus is generally believed to be secondary to underlying lupus glomerulonephritis. ${ }^{1,2}$ Indeed, Rosenbaum $e t a l^{3}$ found that the incidence of hypertension in patients with lupus nephritis increased with both histological activity and loss of renal function. However, Ward and Studenski, ${ }^{4}$ who followed up 160 patients with lupus nephritis for a median of 6.4 years found that hypertension was an important independent risk factor for the development of end stage renal disease and was not merely a surrogate measure of underlying glomerulonephritis. Furthermore there has been a report of a dissociation of hypertension and lupus nephritis in patients with systemic lupus erythematosus, ${ }^{5}$ and it is well known that hypertension may be absent even in patients with aggressive lupus histology and significant renal functional impairment. ${ }^{5}$ This retrospective study was therefore undertaken in order to ascertain the prevalence of hypertension at the onset of clinical lupus nephritis. We also investigated a possible association between hypertension and renal vascular lesions thereof with renal functional impairment. Finally, we searched for a correlation between hypertension and histological class of lupus nephritis.

It was decided to explore these associations on presentation of the disease, as the prognostic importance of hypertension at the onset of nephritis has not been well established. ${ }^{4}$ Furthermore, while the detrimental effect of hypertension on renal function late in the course of lupus nephritis has been recognised, ${ }^{6-9}$ it is nevertheless difficult to draw firm conclusions about its prognostic importance at this stage of the disease, as it may be both a cause and consequence of renal insufficiency. ${ }^{4}$

\section{Patients and methods}

Between 1988 and 1993, all patients who had fulfilled the 1982 American Rheumatism Association Revised Criteria for the classification of systemic lupus erythematosus ${ }^{10}$ and who had undergone renal biopsies at King Edward VIII Hospital were retrospectively assessed. Patients who had no evidence of lupus nephritis according to the World Health Oganisation (WHO) classification ${ }^{11}$ were excluded, as were patients who were on corticosteroid therapy prior to the renal biopsy. None of the remaining patients had any evidence of other identifiable secondary causes of hypertension. A total of 44 patients, (39 women) satisfied these criteria and were selected for the study. The mean age was 33 years (range 15-55); 29

\begin{tabular}{|l|}
\hline $\begin{array}{l}\text { Causes of hypertension in systemic } \\
\text { lupus erythematosus }\end{array}$ \\
\hline - proliferative lupus nephritis \\
- corticosteroid therapy \\
- advanced renal functional impairment
\end{tabular}

Box 1 
regarded as being hypertensive if they had a sitting blood pressure equal to or greater than $140 / 95 \mathrm{mmHg}$ on at least two different occasions. The recorded blood pressure was the mean diastolic pressure of the two determinations. None of the patients was receiving antihypertensive medication at the time of assessment. Hypertension was classified as being mild (diastolic $95-99 \mathrm{mmHg}$ ), moderate $(100-114 \mathrm{mmHg})$ or severe ( $>115 \mathrm{mmHg}$ ). Impaired renal function was defined as a serum creatinine equal to or greater than $120 \mu \mathrm{mol} / \mathrm{l}$, and was graded as mild $(120-200 \mu \mathrm{mol} / 1)$, moderate $(200-$ $350 \mu \mathrm{mol} / \mathrm{l})$ or severe $(>350 \mu \mathrm{mol} / 1)$. The histological classification was obtained from the pathologist's reports which utilise the standard WHO classification of glomerular involvement in systemic lupus erythematosus. ${ }^{11}$ All histology was subsequently re-evaluated by a single senior pathologist who searched specifically for hypertensive renal vascular lesions. The latter were defined as the presence of one or more of the following abnormalities in the arterioles and small arteries: hyperplasia of the media, fibro-elastosis of the intima, and hyalinosis and sclerosis of the vessel wall.

\section{Statistical analysis}

Means and standard deviations were calculated and compared using either the student's unpaired $t$-test for normal distributions, or the Wilcoxon 2-sample test (the non-parametric equivalent), for skewed distributions. The chi-square test was used to compare categorical data. Spearman's correlation coefficient was utilised to determine a possible correlation between the severity of hypertension and both renal function and histological class.

\section{Results}

Hypertension was diagnosed in 17 of the 44 patients $(38 \%)$ at the onset of clinical lupus nephritis. The majority (12 patients) had moderate hypertension. Mild and severe hypertension was present in three and two patients, respectively. The mean age of the hypertensives (35.4 years) did not differ significantly from that of the 27 normotensive patients (32.4 years). The race of the patients did not influence the prevalence of hypertension.

The incidence of impaired renal function was significantly higher in the hypertensives (8/ 17 patients, $47 \%$ ) compared to the normotensives $(5 / 27$ patients, $18.5 \%)(p=0.04)$. The

Table 1 Renal function and blood pressure in patients with and without hypertensive renal vascular lesions

\begin{tabular}{lll}
\hline & $\begin{array}{l}\text { Lesions } \\
\text { present }(n=11)\end{array}$ & $\begin{array}{l}\text { Lesions } \\
\text { absent }(n=33)\end{array}$ \\
\hline Mean diastolic blood pressure $(\mathrm{mmHg})$ & 104.5 & $84.2(\mathrm{p}=0.0001)$ \\
Mean serum creatinine $(\mu \mathrm{mol} / \mathrm{l})$ & 392.7 & $97.0(\mathrm{p}=0.01)$ \\
Prevalence of renal functional impairment $(\%)$ & 63.6 & $18.1(\mathrm{p}=0.01)$ \\
\hline
\end{tabular}

Table 2 WHO histological classification of hypertensives (HT) compared to normotensives (NT). Percentages are given in parentheses

\begin{tabular}{lll}
\hline WHO class & $H T(n=17)$ & $N T(n=27)$ \\
\hline I & - & - \\
II & $2(12)$ & $7(26)$ \\
III & $1(6)$ & - \\
IV & $6(35)$ & $4(15)$ \\
V & $7(41)$ & $16(59)$ \\
VI & $1(6)$ & - \\
\hline
\end{tabular}

mean serum creatinine was also higher in the hypertensives compared to the normotensives: $283.4 \mu \mathrm{mol} / 1$ vs $100.2 \mu \mathrm{mol} / 1$, respectively $(\mathrm{p}=0.02)$. Hypertensive renal vascular lesions were identified in 11 patients, all of whom were hypertensive. Details of renal functional impairment and mean diastolic blood pressure in these patients compared to those without hypertensive renal vascular lesions are described in table 1 . The WHO histological classification of the hypertensives compared to the normotensives is depicted in table 2 . Although there appeared to be a greater prevalence of the milder histological classes in the normotensives ( $26 \%$ class II vs $12 \%$ and $59 \%$ Class V vs $41 \%$ ) and more patients with Class IV histology in the hypertensive group (35\% vs 15\%), these differences were not statistically significant. However, five of the seven hypertensives and six of the 16 normotensives with Class $\mathrm{V}$ histology had proliferative lesions (either Class Vc or Vd).

Proliferative lesions (excluding patients with Class II histology) were therefore present in 12 of the 17 hypertensives $(70 \%)$ compared to only 10 of the 27 normotensives (37\%), $p=0.03$. There was no relationship between race and the prevalence of any of the histological classes. In the hypertensive group there was no correlation between the severity of hypertension and either renal function $(r=0.09)$ or histological class $(r=0.08)$.

\section{Discussion}

Systemic lupus erythematosus was previously regarded as an uncommon disease in Africa. $^{12-14}$ Subsequently, however, perhaps consequent upon an increased medical awareness and wider use of screening tests, ${ }^{15}$ it has been recognised more frequently in Southern Africa. ${ }^{15-17}$ Furthermore, the disease has serious consequences in South African Blacks, being associated with a high mortality. ${ }^{18}$ Renal disease in our patients with systemic lupus erythematosus is common, with a reported prevalence of $65 \%$ and is a leading cause of mortality. ${ }^{18} \mathrm{~A}$ recent study conducted at our institution reported a mortality of $35 \%$ in patients with lupus nephritis. ${ }^{15}$

This high mortality, largely due to the development of end stage renal failure, ${ }^{15}$ is of particular concern to us, as the majority of our patients are socio-economically deprived Blacks in whom renal replacement therapy is not feasible because most of them reside either 
in a rural environment or in peri-urban settlements lacking basic ablution and sanitary facilities. ${ }^{19}$

The role of hypertension at the onset of clinical lupus nephritis as a risk factor for progression to end-stage renal disease is controversial. ${ }^{5}$ Ward and Studenski ${ }^{4}$ found that hypertension and cigarette smoking at the onset of nephritis were important, independent risk factors for progression to end-stage renal disease. Appel et al, ${ }^{8}$ however, reported that hypertension at the time of the initial renal biopsy did not significantly influence renal or patient survival. Similarly, Nossent et $a l^{20}$ in a prospective study, found no relation between the presence of hypertension and severity of disease course. They commented, however, that the early detection and effective treatment of arterial hypertension in their patients probably eliminated its contribution to subsequent renal damage.

The prevalence of hypertension at the onset of clinical lupus nephritis in our patients was $38 \%$, and was not influenced by age or race. This is identical to the prevalence reported by Ward and Studenski, ${ }^{4}$ and is considerably higher than the prevalence of hypertension in our adult Black and Indian population, viz; $25 \%$ and $14.2 \%$, respectively. ${ }^{21}$ None of our patients were receiving corticosteroids at the time of analysis and the incidence of hypertension would be expected to increase with the evolution of the disease and initiation of corticosteroids. Even at this early stage of the illness, however, hypertension appeared to be functionally significant in that both the incidence of renal functional impairment and mean serum creatinine levels were significantly higher in the hypertensives than in the normotensives. Furthermore, the presence of hypertensive renal vascular lesions identified a highrisk subgroup of patients who had a higher incidence of renal functional impairment and worse renal function than the hypertensive group as a whole (table 1). All our patients with hypertensive renal vascular lesions were hypertensive. We were unable to demonstrate a correlation between hypertension and histological class of lupus nephritis (table 2).

Nevertheless, proliferative lesions (including patients with WHO Class Vc and Vd histology and excluding those with Class II histology) were significantly more prevalent in the hypertensives compared to the normotensives, suggesting that aggressive lupus histology may play a role in the pathogenesis of hypertension in systemic lupus erythematosus. In this context, Appel $e t a l^{B}$ reported that their patients with WHO Class IIa and IIb histology had a more favourable renal and patient survival at five and 10 years than did patients in the other WHO classes (III, IV and V). They noted that the long-term prognosis for Class $\mathrm{V}$ patients was less favourable than originally suggested. From the point of view of hypertension, however, the presence of two hypertensive patients with Class II histology and four normotensives with Class IV histology (table 2) suggests that factors other than lupus histology must be involved in the pathogensis of hypertension in these pa-

\section{Summary/learning points}

- the prevalence of hypertension at the onset of clinical lupus nephritis was $38 \%$

- the incidence of renal functional impairment and mean serum creatinine levels were higher in the hypertensives than the normotensives

- patients with hypertensive renal vascular lesions represented a high-risk subgroup with a higher incidence of renal functional impairment and worse renal function than the hypertensive group as a whole

- severe (proliferative) lupus histology was more prevalent in hypertensive patients

- in addition to immunosuppressive therapy, effective treatment of hypertension in patients with early lupus nephritis is essential as hypertension is an important, potentially modifiable, risk factor for progression to end stage renal failure in these patients

\section{Box 2}

tients. Finally, in the hypertensive group the absence of a correlation between the severity of hypertension and both renal function and histological class is of doubtful significance as there were only 17 patients in this group.

As discussed previously lupus nephritis in our patients with systemic lupus erythematosus is common ${ }^{18}$ and is associated with significant mortality, with approximately $25 \%$ of patients dying from end-stage renal failure. ${ }^{15}$ As we are unable to support many of these patients on renal replacement therapy programmes it is important to be able to modify the disease at an early stage. In addition to the adverse prognosis associated with the more severe histological forms of lupus nephritis (especially WHO Class IV and high activity and chronicity indices), ${ }^{6,22-24}$ arterial hypertension is also an important, potentially modifiable risk factor for progression to end-stage renal disease. ${ }^{4,5} \mathrm{We}$ are unable to draw any conclusions about the prognostic significance of hypertension at the onset of clinical lupus nephritis in our patients as this would require a longer period of observation. Nevertheless we were able to demonstrate an association between hypertension and impaired renal function on presentation. Furthermore, the observation that the presence of hypertensive renal vascular lesions identified a subgroup of patients with significantly worse renal function suggests that hypertension is functionally significant at an early stage of lupus nephritis. We therefore believe that, in addition to appropriate immunosuppressive therapy, early and effective treatment of arterial hypertension in patients with early lupus nephritis is essential to prevent deterioration of renal function.

The authors acknowledge Ms Eleanor Gouws of the Biostatistics Department of the Medical Research Council for her assistance with the statistical analysis, and Mrs D Mackrory for her secretarial assistance. 
1 Budman DR, Steinberg AD. Hypertension and renal disease in systemic lupus erythematosus. Arch Intern Med disease in systemic lup

2 Walker WG, Solez K. Renal involvement in disorders of connective tissue. In: Strauss, Welt, eds, Disease of the kidney, 3rd edn. Boston: Little, Brown and Co, 1979; pp $1259-88$.

3 Rosenbaum JL, Akhtar M, Kramer MS. Evaluation of clearance studies in lupus nephritis. Clin Nephrol 1974; 2: $47-51$.

4 Ward MM, Studenski S. Clinical prognostic factors in lupus nephritis. The importance of hypertension and smoking. Arch Intern Med 1992; 152: 2082-8.

5 Petrin J, Rozman B, Dolenc P, et al. The dissociation of arterial hypertension and lupus glomerulonephritis in arterial hypertension and lupus glomerulonephritis in systemic
$108-12$

6 Baldwin DS, Lowenstein J, Rothfield NF, Gallo G, McCluskey RF. The clinical course of the proliferative and membranous forms of lupus nephritis. Ann Intern Med 1970; 7: 929-42.

7 Baldwin DS, Gluck MC, Lowenstein J, Gallo GR. Lupus nephritis: clinical course as related to morphologic forms and their transitions. Am $\mathcal{F}$ Med 1977; 62: 12-30.

8 Appel GB, Cohen DJ, Pirani CL, Meltzer J, Estes D. Longterm follow-up of patients with lupus nephritis: a study based on the classification of the World Health Organization. Am $\mathcal{f}$ Med $1987 ; 83: 877-85$.

9 Leaker B, Fairley KF, Dowling J, Kincaid-Smith P. Lupus nephritis: clinical and pathological correlation. $Q \mathcal{f}$ Med nephritis: clinical

10 Tan EM, Cohen AS, Fries JF, et al. The 1982 revised criteria for the classification of systemic lupus erythematosus. Arthritis Rheum 1982; 25: 1271 - 7.

11 Churg J, Sobin LH. Renal disease classification and atlas of glomerular disease. Tokyo: Igaku-Shoin, 1982; pp 127-49. 2 Shaper AG. Systemic lupus erythematosus. E Afr Med 7 1961; 31: $134-44$.

13 Greenwood BM. Autoimmune disease and parasitic infections. Lancet 1968; ii: $380-2$
14 Jessop S, Meyers OL Systemic lupus erythematosus in Cape Town. S Afr Med f 1973; 47: 222-5.

15 Seedat YK, Parag KB, Ramsaroop R. Systemic lupus erythematosus and renal involvement. A South African experience. Nephron 1994; 66: 426-30.

16 Taylor HG, Stein CM. Systemic lupus erythematosus in Zimbabwe: Ann Rheum Dis 1986; 45: 645-8.

17 Dessein PHMC, Gledhill RF, Rossouw DS. Systemic lupus erythematosus in Black South Africans. S Afr Med F 1988; 74: $387-9$.

18 Moody GM, Parag KB, Nathoo BC, Pudifin DJ, Duursma J, Seedat YK. High mortality with systemic lupus erythematosus in hospitalized African Blacks. Br $\mathcal{f}$ Rheumatol 1994; 33: $1151-3$.

19 Naiker IP, Naicker S, Botden L, Haffejee AA, Seedat YK. Continuous ambulatory peritoneal dialysis - A South Continuous ambulatory peritoneal dialysis - A South

20 Nossent JC, Bronsveld W, Swaak AJG. Systemic lupus eythematosus. III. Observations on clinical renal involvement and follow up of renal function: Dutch experience with 110 patients studied prospectively. Ann Rheum Dis 1989 ; 48: $810-6$.

21 Seedat YK. Race, environment and blood pressure: the South African experience. 7 Hypertens 1993; 1: 7-12.

22 Nossent HC, Henzen-Logmans SC, Vroom JM, Berden JHM Swaak TJG. Contribution of renal biopsy data in predicting outcome in lupus nephritis: analysis of 116 predicting outcome in lupus nephritis:

23 Austin HA, Muenz IR, Joyce KM, Antonovych H, Balow JF. Diffuse proliferative lupus nephritis: identification of JF. Diffuse proliferative lupus nephritis: identification of specific pathological featur

24 Schwartz MM, Bernstein J, Hill GS, Holley K, Phillips FA and the Lupus Nephritis Collaborative Study Group. Predictive value of renal pathology in diffuse proliferative lupus glomerulonephritis. Kidney Int 1989; 36: 891-6. 